East African Medical Journal Vol. 77 No. 9 September 2000

USE OF A SIMPLE PAIN MODEL TO EVALUATE ANALGESIC ACTIVITY OF IBUPROFEN VERSUS PARACETAMOL

I. Lala, P. Leech, L. Montgomery, Visiting Elective Medical Students, University of Alberta and K. Bhagat, BSc, Msc, PhD, MBChB, DCH, DCardiol, FESC, Department of Clinical Pharmacology, University of Zimbabwe, P.O. Box A178, Avondale, Harare, Zimbabwe.

Request for reprints to: Prof. K. Bhagat, Department of Clinical Pharmacology, Health Science Building, University of Zimbabwe, P.O. Box A178, Avondale, Harare, Zimbabwe.

\title{
USE OF A SIMPLE PAIN MODEL TO EVALUATE ANALGESIC ACTIVITY OF IBUPROFEN VERSUS PARACETAMOL
}

\section{LALA, P. LEECH, L. MONTGOMERY and K. BHAGAT}

\begin{abstract}
Objective: To evaluate the analgesic activity of ibuprofen against paracetamol using a simple pain model.

Design: A double-blind study.

Setting: Twenty general practitioners in Harare, Zimbabwe.

Patients: Adults with acute sore throat of a maximum of two days' duration.

Interventions: One hundred and thirteen patients with acute pain associated with tonsillopharyngitis randomly received either $400 \mathrm{mg}$ ibuprofen or $1000 \mathrm{mg}$ paracetamol. The study design included repeated administration up to 48 hours to assess tolerability.

Main outcome measures: At hourly intervals for six hours after the first dose of treatment, the patients evaluated pain intensity on swallowing, difficulty in swallowing and global pain relief according to visual analogue scales.

Results: Ibuprofen $400 \mathrm{mg}$ was significantly more effective than paracetamol $1000 \mathrm{mg}$ in all three ratings, at all time-points for pain intensity and difficulty in swallowing, and from two hours onwards for pain relief. There were no serious adverse effects and no statistically significant difference in the incidence of adverse effects in the two treatment groups.

Conclusions: Sore throat pain provided a sensitive model to assess the analgesic efficacy of class I analgesics and discriminated between the analgesic efficacy of ibuprofen and paracetamol.
\end{abstract}

\section{INTRODUCTION}

Various clinical conditions have been used as models to assess pain relief following the administration of different analgesics. These models include dental pain, dysmenorrhoea, osteoarthritis of the knee and, more recently, sore throat pain. Sore throat, associated with tonsillitis, is a frequent complaint, and has been shown to provide a sensitive model for the evaluation of class I analgesics $(1,2)$. In these studies patients' descriptions of their pain were highly correlated with the physicians' assessments of the severity of sore throat. The sore throat model has been successfully employed in clinical trials to distinguish between different analgesic agents and placebo, and between different doses of an analgesic(4).

The sore throat model consisted of five subscales: pain intensity, relief of pain, change in pain, pain severity description (mild, moderate and severe), and maximum reduction in pain (no reduction to complete reduction). The scales also included two sensory qualities of sore throat pain (swollen throat and difficulty in swallowing) and the assessment period was set at six hours postdose allowing patients to complete the assessments between three to six hours at home. Furthermore the addition of a pain subscale (a $200 \mathrm{~mm}$ visual analogue scale(VAS)) was added to discriminate between the analgesic effects of aspirin and an aspirin/caffeine combination.
Two extensively used analgesics in the treatment of sore throat pain are ibuprofen and paracetamol. Despite their wide use, to our knowledge there are no studies that have compared their relative analgesic efficacy using a sore throat model.

The present randomised, double-blind, multicentre, parallel group comparative study was performed to validate the sore throat model described.

\section{MATERIALS AND METHODS}

Study participants: In this twenty GP practice, primary care study, adults consulting their general practitioner with acute sore throat (onset within two days) were recruited into the study. Patients were eligible to enter the study if they had at least moderately severe throat pain (a score greater than $50 \mathrm{~mm}$ on a $100 \mathrm{~mm}$ VAS with end-points no pain and very intense pain) associated with an obvious diagnosis of tonsillo-pharyngitis (a score of 4 or more on the 10-point tonsillo-pharyngitis scale).

Patients excluded from the study were: those who had a history of hypersensitivity to ibuprofen, aspirin, paracetamol or any other NSAID or any contraindication to treatments; those who had used any local treatment such as an anaesthetic spray within two hours, any analgesic treatment within six hours and/ or any anti-inflammatory treatment within three days prior to the initial consultation; those who had mouth breathing as a result of severe nasal congestion (mouth breathing could alter the symptomatology and natural history of acute sore throat thus confounding the results). 
Patients should not have received any antibiotic treatment within the week prior to the initial consultation. However, patients could be treated with antibiotics, provided that this medication was not started until at least six hours after the first dose of study treatment. All patients provided written consent before participating in the study.

Study assessments: The severity of sore throat was assessed by the patient, using the following three indices: pain on swallowing according to a continuous $100 \mathrm{~mm}$ visual analogue scale (VAS) with end-points of no pain to very intense pain; relief of sore throat pain using a numeric scale (end-points $0 \%$ relief to $100 \%$ relief; in blocks of $10 \%$ ) and; difficulty in swallowing using a VAS of $100 \mathrm{~mm}$ (end-points of no difficulty to very great difficulty).

These assessments were made at home by the patient every hour for six hours following the first dose of treatment. In addition, a global evaluation of treatment efficacy was made after 48 hours at the end of the study by the patients according to 4-point verbal scales (not satisfied, a little satisfied, mostly satisfied, very satisfied).

The severity of sore throat was also assessed by the investigator at both the screening and final visits, using the tonsillo-pharyngitis score, an index that takes into account as a sum of ratings (on a 0 to 2-point scale) the intensity of each of five clinical features of tonsillo-pharyngitis (oral temperature, oropharyngeal coloration, oropharyngeal erythaema, cervical adenopathy and cervical adenitis). A global evaluation of treatment efficacy was also performed at the end of the study by investigators according to 4-point verbal scales (none, moderate, good, very good). All adverse effects during the study period were collected.

Study treatments: Patients meeting admission criteria were allocated by a computer-generated randomisation code to receive either 400mg ibuprofen or $1000 \mathrm{mg}$ paracetamol under doubleblind conditions. To maintain blinding, a double-dummy design was used. All treatments were supplied by Knoll, UK. Each treatment dose comprised either one tablet of ibuprofen $400 \mathrm{mg}$ and two capsules of paracetamol placebo or one tablet of ibuprofen placebo and two capsules of paracetamol 500mg. Treatment was to be taken three times daily for up to two days with a minimum of six hours between each dose.

Study design: The first dose of study treatment was administered in the GP's office, after which patients were discharged. Patients evaluated their throat pain every hour over the next six hours, during which time they were to take no further doses of study medication or use any other treatment. If antibiotic therapy was considered necessary, this was only to be started after six hours following the first dose of analgesic. Patients recorded the results of their hourly assessments on a diary card. This card was also used to record the times of subsequent treatment doses, as well as the occurrence of any adverse effects, over the rest of the study period (two days).

Patients returned for a final consultation after 48 hours, at which time tonsillo-pharyngitis scores and global assessments of efficacy were recorded.

Statistical methods: Observed differences between the two treatment groups in pain or swallowing were tested for significance ( $p<0.05$ ) using a 2-factor analysis of variance (ANOVA) with centre and treatment as main fixed effects.

The evolution of pain on swallowing was analysed as the absolute difference from baseline (pain intensity difference PID), both over one hour (h) one to six hours using a 2-factor ANOVA with repeated measures and at individual time-points using a 2-factor ANOVA. The sum of pain intensity differences at six hours (SPID) was analysed using a 2-factor ANOVA. The difficulty in swallowing and global relief scales were analysed similarly as the absolute difference from baseline (1h to $6 \mathrm{~h}$ ) and at each time-point, and as the sum of the difference scores at 6 hours (analogous to PID and SPID), but using a 1-factor ANOVA in all cases.

If patients took any medication within the first six hours, pain assessments after treatment intake were replaced with the score at the time of re-medication using the last observationcarried-forward method. Time to re-medication, the final tonsillopharyngitis score and the global evaluation of treatment efficacy were compared between groups using a Wilcoxon test.

The evolution of pain on swallowing was considered as the primary efficacy variable in this study and intent-to-treat analyses were supported by analyses using a per-protocol population.

The tolerability analyses were carried out on all patients who took at least one dose of study medication. The number of patients who experienced at least one adverse effect during the study was compared between groups using a $\mathrm{Chi}^{2}$ test.

\section{RESULTS}

Study population: Fifty seven patients were randomly assigned to the ibuprofen treatment group and 56 to the paracetamol group. All 113 patients took at least one dose of study medication and 110 patients (55 in each group) completed the study.

Nine patients had major deviations from the protocol during the study that necessitated their exclusion from the per-protocol analysis of the primary efficacy variable. Seven patients were treated with antibiotic medication within three hours of the first dose of study treatment, one patient in the ibuprofen group took paracetamol at days 0 and one of the study, and one patient had pain assessment times that deviated by more than 30 minutes from the protocol-defined schedule. The per-protocol population thus contained 104 patients (52 in each group). There were no significant differences between the two groups at baseline assessment, particularly in the pertinent clinical features (Table 1).

Table 1

Clinical features of the study population with tonsillopharyngitis at day 0

\begin{tabular}{lll}
\hline Feature (mean $\pm \mathrm{SD})$ & \multicolumn{2}{c}{ Treatment group } \\
& $\begin{array}{l}\text { Ibuprofen } 400 \mathrm{mg} \\
(\mathrm{n}=57)\end{array}$ & $\begin{array}{l}\text { Paracetamol } \\
(1000 \mathrm{mg}) \\
\mathrm{n}=56\end{array}$ \\
\hline Age & $37.5(19-58)$ & $37.6(20-61)$ \\
Gender: Males & $28(49)$ & $23(41)$ \\
Duration of sore throat & $1.1 \pm 0.48$ & $1.1 \pm 0.56$ \\
$\begin{array}{l}\text { Tonsillopharyngitis } \\
\text { score (10) }\end{array}$ & $6.4 \pm 1.2$ & $6.4 \pm 1.1$ \\
$\begin{array}{l}\text { Pain intensity on } \\
\text { swallowing (mm) }\end{array}$ & $76.6 \pm 14.3$ & $73.3 \pm 11.5$ \\
$\begin{array}{l}\text { Difficulty in swallowing }(\mathrm{mm}) \\
73.9 \pm 14.3\end{array}$ & $71.8 \pm 14.6$ \\
\hline
\end{tabular}

All between group differences were non-significant $(p>0.1)$

Efficacy: The sore throat model used in this study discriminated between the analgesic efficacy of $400 \mathrm{mg}$ ibuprofen and $1000 \mathrm{mg}$ paracetamol. Ibuprofen was 
Table 2

Absolute changes from baseline (mean $\pm S D$ ) in pain measures at each hour over the first six hours after the first treatment

\begin{tabular}{|c|c|c|c|c|c|}
\hline \multirow[t]{2}{*}{ Time (h) } & \multicolumn{3}{|c|}{ Pain on swallowing (mm) PID } & \multicolumn{2}{|c|}{ Difficulty in swallowing (mm) PID } \\
\hline & Ibuprofen & Paracetamol & $\mathrm{P}$ value $(\mathrm{A})$ & Ibuprofen & Paracetamol \\
\hline 1 & $-12.5 \pm 16.2$ & $-4.2 \pm 8.0$ & $<0.001$ & $-11.8 \pm 14.9$ & $-5.1 \pm 9.8$ \\
\hline 2 & $-22.5 \pm 14.3$ & $-10.9 \pm 14.3$ & $<0.001$ & $-21.5 \pm 20.0$ & $-12.4 \pm 15.1$ \\
\hline 3 & $-29.2 \pm 19.4$ & $-15.5 \pm 14.3$ & $<0.001$ & $-28.8 \pm 21.1$ & $-17.0 \pm 15.9$ \\
\hline 4 & $-30.0 \pm 19.3$ & $-17.3 \pm 15.2$ & $<0.001$ & $-29.2 \pm 20.2$ & $-18.5 \pm 17.4$ \\
\hline 5 & $-28.9 \pm 20.5$ & $-15.2 \pm 12.6$ & $<0.001$ & $-27.8 \pm 21.1$ & $-16.3 \pm 16.4$ \\
\hline 6 & $-28.8 \pm 22.1$ & $-14.0 \pm 14.3$ & $<0.001$ & $-28.0 \pm 22.7$ & $-15.4 \pm 18.5$ \\
\hline
\end{tabular}

(a) Two factor ANOVA

(b) One factor ANOVA
NS=non-significant

$\mathrm{PID}=$ Pain intensity difference significantly superior to paracetamol in all three pain measurement scales used.

Ibuprofen resulted in statistically significantly greater reductions in the intensity of pain on swallowing (the primary efficacy variable) at each time-point over the six hour evaluation period (Table 2), as well as a statistically significant difference in the overall effect, that is, the sum of changes from baseline to 6 hours postdose (Table 3 ).

Ibuprofen reduced the difficulty in swallowing at each time-point over the six-hour evaluation period significantly more than paracetamol (Table 2). Overall effect, that is, the sum of reductions in the difficulty in swallowing from baseline to six hours postdose, was significantly greater with ibuprofen (Table 3 ). Ibuprofen was significantly superior to paracetamol in the improvement in pain relief at time-points h2 to h6 (Table 2 ) as well as in the overall effect (Table 3).

There were no significant differences between treatment groups in the time to re-medication or in the improvement in tonsillo-pharyngitis score at the end of the study. The median time for remedication in both groups was 360 minutes, with paracetamol showing a wider variation.

Table 3

Sum values of changes in pain measures over the first six hours after treatment

\begin{tabular}{llll}
\hline & \multicolumn{2}{c}{ Treatment group } & \\
Variable & Ibuprofen & Paracetamol & p value \\
& $(\mathrm{n}=57)$ & $(\mathrm{n}=56)$ & \\
\hline
\end{tabular}

Pain on swallowing

$\begin{array}{lllr}\text { (SPID) } & -151.7 \pm 102.2 & -77.1 \pm 68.0 & <0.001(\mathrm{a}) \\ \text { Difficulty in swallowing } & -147.2 \pm 106.0 & -84.6 \pm 82.6 & <0.001(\mathrm{~b}) \\ \text { Pain relief } & +215.1 \pm 130.5 & +154.5 \pm 101.7 & 0.007 \text { (b) }\end{array}$

(a) Two factor ANOVA

(b) One factor ANOVA

$\mathrm{SPID}=$ sum of pain intensity differences
Table 4

Adverse effects reported during the study (a)

\begin{tabular}{|c|c|c|}
\hline & \multicolumn{2}{|c|}{ Treatment group } \\
\hline & $\begin{array}{l}\text { Ibuprofen } 400 \mathrm{mg} \\
(\mathrm{n}=57)\end{array}$ & $\begin{array}{c}\text { Paracetamol } 1000 \mathrm{mg} \\
\qquad(\mathrm{n}=56)\end{array}$ \\
\hline $\begin{array}{l}\text { Patient with at least one } \\
\text { adverse effect (No }(\%))\end{array}$ & $12(21.1)$ & $11(19.6)$ \\
\hline Total number of effects & 17 & 15 \\
\hline Diarrhoea & 2 & 1 \\
\hline Oesophagitis & 1 & - \\
\hline Abdominal pain & 8 & 1 \\
\hline Vomiting & 1 & \\
\hline Nausea & 1 & - \\
\hline Insomnia & 1 & - \\
\hline Hot flushes & 1 & - \\
\hline Somnolence & 1 & - \\
\hline Dizziness & 1 & - \\
\hline Flatulence & - & 1 \\
\hline Rhinitis & - & 3 \\
\hline Asthenia & - & 1 \\
\hline Headache & - & 2 \\
\hline Cough & - & 2 \\
\hline Fever & - & 1 \\
\hline
\end{tabular}

(a) Between group comparison of patients with at least one adverse effect was non-significant $(\mathrm{p}=0.85)$

Tolerability: Twelve (21\%) patients in the ibuprofen group and eleven $(20 \%)$ patients in the paracetamol group experienced at least one adverse effect $(p=0.85)$. Most adverse effects with ibuprofen were gastrointestinal disturbances (Table 4), but were all of mild/moderate intensity and did not lead to discontinuation of study treatment, except for one case of severe gastralgia in the ibuprofen group, which was not associated with serious gastrointestinal complications. The most frequent adverse effects with paracetamol were rhinitis, headache and cough.

\section{DISCUSSION}

This clinical study provides substantiation that the sore throat pain model is a sensitive assay of analgesic activity and confirms the superiority of ibuprofen over paracetamol. 
Sore throat pain appears to be a valid model for nociceptive pain with a demonstrated ability to discriminate the oral analgesics aspirin, paracetamol and ibuprofen from placebo. Like dental pain models $(5,6)$, this model enables significant analgesic effects to be demonstrated using relatively small sample sizes. The model also appears able to discriminate between different active agents, and thus fulfils scientific criteria for an acceptable pain model(1).

Our study highlighted the clinically relevant differences in analgesic efficacy between a single dose of ibuprofen 400mg and paracetamol 1000mg. Differences between the treatments were assessed using three pain measures: intensity of pain on swallowing, "difficulty in swallowing', a frequent complaint correlating highly with pain intensity and serving as a sensitive indicator of pharmacological response and; an assessment of relief of pain using a 10-block numerical scale

Upside assay sensitivity was highlighted by comparing the PID scores for all three measures between ibuprofen 400mg and paracetamol $1000 \mathrm{mg}$ at each hour over the six hours immediately following the first dose of treatment. Statistically significant differences between treatments were detected from as early as one hour following treatment for pain intensity and dysphagia, and from two hours onwards for pain relief. The various subjective assessments of characteristics of pain (dysphagia) and pain relief thus demonstrated analgesic discrimination and yielded consistent statistically significant results that agreed with the result obtained using the conventional pain intensity scale.

Although the study was randomised and carried out in accordance with good clinical practice, it had some limitations, which included: primary assessment of efficacy only after the first dose; involvement of many GP practices which have confounded the outcome of the treatment although no statistical interactions between treatment and centres were detected; and the severity of the disease was at least moderate. These factors may, in certain circumstances, affect the generalisability of the study results.

The differences between treatments were both statistically and clinically significant. Ibuprofen provided at least ten per cent more pain relief than paracetamol as measured by the VAS. This study has also shown that all assessments can be made at home by the patients. This makes the model appropriate for assessing the analgesic activity of over-the-counter medications.

Practising physicians should consider ibuprofen as an effective alternative to paracetamol in the treatment of sore throat pain. The superiority of ibuprofen in the treatment of sore throat pain may be due to its superior inhibition of peripheral prostaglandins. At elevated doses (more than 1200mg per day), ibuprofen is known to have anti-inflammatory activity. However, the evidence of this anti-inflammatory activity at lower doses is questionable. The sore throat model should be considered by researchers as a sensitive assay in measuring analgesic and potentially anti-inflammatory activities of medications.

In conclusion, this study confirmed that the sore throat model is a sensitive assay in measuring relative analgesic efficacy of commonly used medications. Ibuprofen provided very rapid relief of painful symptoms with a significant advantage over paracetamol from as early as just one hour following a single dose of treatment. Both treatments were well tolerated and any adverse effects were generally mild or moderate and did not require discontinuation of therapy.

\section{ACKNOWLEDGEMENTS}

We are grateful to all the general practitioners that we involved in the study in addition to those that did not participate but provided us with useful advice and assistance throughout the course of the investigation.

\section{REFERENCES}

1. Schachtel B.P. Sore throat pain. In: Portenoy M.M., Laska E. editors. Advances in pain research and therapy. Vol 18. New York: Raven Press Ltd, 1991; 393-407

2. Bertin L., Pons G. and d'Athis P. Randomised, double-blind, multicentre, controlled trial of ibuprofen versus acetaminophen (paracetamol) and placebo for treatment of symptoms of tonsillitis and pharyngitis in children. J. Paediat. 1991; 119:811-4

3. Schachtel B.P., Fillingim J.M. and Beiter D.J. Subjective and objective features of sore throat. Arch Intern Med 1984; 144:497500

4. Cooper S.A. and Beaver W.T. A model to evaluate mild analgesics in oral surgery outpatients. Clin Pharmacol Ther. 1976;20:241-50

5. Cooper S.A. Five studies on ibuprofen for postsurgical dental pain. Amer J Med. 1984; 77: Suppl:70-7. 